\title{
New Views on Dark Matter from Emergent Gravity
}

\author{
Sichun Sun ${ }^{1, \star}$ and Yun-Long Zhang ${ }^{2,}$ \\ ${ }^{1}$ Center for Theoretical Sciences, National Taiwan University, Taipei 10617, Taiwan \\ ${ }^{2}$ Asia Pacific Center for Theoretical Physics, Pohang 790-784, Korea
}

\begin{abstract}
We discuss a scenario that apparent dark matter comes from the induced gravity in the $(3+1)$ dimensional spacetime, which can be embedded into one higher dimensional flat spacetime. The stress tensor of dark energy and dark matter is identified with the Brown-York stress tensor on the hypersurface, and we find an interesting constraint relation between the dark matter and dark energy density parameter and baryonic density parameter. Our approach may show a new understanding for Verlinde's emergent gravity from higher dimensions. We also comment on some phenomenological implications, including gravitational wave solutions and MOND limit.
\end{abstract}

\section{A Toy Model for the late Universe}

Inspired by the emergent gravity models proposed by Verlinde in [1,2], we propose the induced gravity from higher dimensional spacetime which gives rise to the similar mechanism. We will modify the Einstein field equation in 3+1 dimensional spacetime at the cosmological scale,

$$
R_{\mu v}-\frac{1}{2} R g_{\mu v}+\frac{H_{0}}{c}\left(\mathcal{K} g_{\mu v}-\mathcal{K}_{\mu v}\right)=\frac{8 \pi G}{c^{4}} T_{\mu \nu},
$$

where $H_{0}$ is the Hubble constant. $G$ is the newton gravitational constant. $c$ is the speed of light. $T_{\mu \nu}$ is the stress tensor for normal matters. Alternatively, we can also put the extra terms on the right hand side, which adds the extra contribution to the stress tensor

$$
\mathcal{T}_{\mu \nu} \equiv-\frac{H_{0} c^{3}}{8 \pi G}\left(\mathcal{K} g_{\mu \nu}-\mathcal{K}_{\mu \nu}\right)
$$

It is just the Brown-York stress tensor induced from higher dimensional space time, and we explain it as the apparent dark sectors. We are going to show that stress tensor $\mathcal{T}_{\mu \nu}$, which is holographic in nature [4] can provide dark energy and dark matter effects.

We consider the $\Lambda \mathrm{CDM}$ Universe, in which the universe contains a positive cosmological constant $\Lambda$ contribution to the dark energy with component $\Omega_{\Lambda}$, cold dark matter density parameter $\Omega_{D}$, and Baryon density parameter $\Omega_{B}$. They satisfy $\Omega_{D}+\Omega_{B}+\Omega_{\Lambda} \simeq 1$. Based on eq.(1) and constraints from the consistent embedding, we obtain an interesting constraint relation of the uniform dark matter density,

$$
\mathrm{CSZ}: \quad \Omega_{D}^{2}=\frac{1}{2} \Omega_{\Lambda}\left(\Omega_{D}-\Omega_{B}\right) .
$$

${ }^{\star}$ Corresponding Author and Speaker: sichunssun@gmail.com. Proceeding for the 13th International Conference on Gravitation, Astrophysics, and Cosmology the 15th Italian-Korean Symposium on Relativistic Astrophysics: A Joint Meeting. Parallel Talk on July 4, 2017, Seoul, Korea.
We will show exactly how to derive this equation in the next section. Let us compare with the constraint relation in Verlinde's emergent gravity [2],

$$
\text { Verlinde: } \Omega_{D}^{2}=\frac{4}{3} \Omega_{B} \text {. }
$$

We take some parameters from the current $\Lambda \mathrm{CDM}$ universe from observation[3], with a bit priori choice as following

$$
\Omega_{\Lambda}=0.685, \quad \Omega_{D}=0.265, \quad \Omega_{B}=0.050 .
$$

Comparing our formula (3) with Verlinde's (4), we obtain

$$
\begin{aligned}
\Delta_{V} & \equiv \Omega_{D}^{2}-\frac{4}{3} \Omega_{B} \simeq 0.36 \%, \\
\Delta_{C S Z} & \equiv \Omega_{D}^{2}-\frac{1}{2} \Omega_{\Lambda}\left(\Omega_{D}-\Omega_{B}\right) \simeq-0.34 \% .
\end{aligned}
$$

We will show that although our relation hold as well as Verlinde's, there are still some differences in approximation.

Constraints From Consistent Embedding — Similar to the formula in (1), let us write down the Einstein equation in $d$ dimensional spacetime as

$$
R_{\mu v}-\frac{1}{2} R g_{\mu \nu}=\kappa_{d}\left(T_{\mu \nu}+\mathcal{T}_{\mu v}\right),
$$

with $\mu, v=0,1, \ldots,(d-1)$, and $\kappa_{d}=8 \pi G_{d} / c^{4} . T_{\mu \nu}$ is the stress tensor of normal matters, and $\mathcal{T}_{\mu \nu}$ is the effective dark sectors of our universe, which can include both of the dark energy and dark matters. The trace of above equation gives the Ricci scalar

$$
R=-\frac{2 \kappa_{d}}{d-2}(T+\mathcal{T})
$$

Now we assume that the geometry with metric $g_{\mu \nu}$ can be embedded into one higher dimensional spacetime, as a 
hypersurface with the normal vector $\mathcal{N}^{M}$, and the indices $M, N=0,1, \ldots, d$. We can define the induced metric on the hypersurface $g^{M}{ }_{N}=\eta^{M}{ }_{N}-\mathcal{N}^{M} \mathcal{N}_{N}$ as well as the extrinsic curvature

$$
\mathcal{K}_{\mu v} \equiv g_{\mu}{ }^{M} g_{v}{ }^{N} \nabla_{(M} \mathcal{N}_{N)}
$$

$\mu, v$ are the indices on the hypersurface, which depend on the coordinate choices.

On the other hand, the Hamiltonian constraint equations of the hypersurface lead to

$$
R=\mathcal{K}^{2}-\mathcal{K}_{\mu \nu} \mathcal{K}^{\mu \nu}-2 G_{M N}^{(d+1)} \mathcal{N}^{M} \mathcal{N}^{N}
$$

Now we introduce the following Brown-York stress tensor,

$$
\mathcal{T}_{\mu \nu}=-\frac{1}{\kappa_{d+1}}\left(\mathcal{K} g_{\mu \nu}-\mathcal{K}_{\mu \nu}\right)
$$

and $\kappa_{d+1}$ is the Einstein's constant in $d+1$ dimension. Notice that in the above definition, there is a minus sign comparing to the usual Brown-York formula [4]. Replacing the external curvature by the Brown-York stress tensor, the Hamiltonian constraint relation (11) becomes

$$
\frac{\mathcal{T}^{2}}{d-1}-\mathcal{T}_{\mu \nu} \mathcal{T}^{\mu \nu}=\frac{R+2 G_{M N}^{(d+1)} \mathcal{N}^{M} \mathcal{N}^{N}}{\left(\kappa_{d+1}\right)^{2}} .
$$

Even though there are matters in the late universe, we require them to be localized on the hypersurface, such that we still have $G_{M N}^{(d+1)} \mathcal{N}^{M} \mathcal{N}^{N}=T_{M N}^{(d+1)} \mathcal{N}^{M} \mathcal{N}^{N}=0$ in (11). Then plug (9) into (13), we have

$$
\frac{\mathcal{T}^{2}}{d-1}-\mathcal{T}_{\mu \nu} \mathcal{T}^{\mu \nu}=-\frac{2 \kappa_{d}}{\left(\kappa_{d+1}\right)^{2}} \frac{1}{d-2}(T+\mathcal{T})
$$

Pure De Sitter Spacetime. - Firstly we set that the normal matters in the Einstein equation (8) vanish, $T_{\mu \nu}=0$. As a warm up, let us consider the $d$ dimensional de-Sitter spacetime,

$$
\mathrm{d} s^{2}=-c^{2} \mathrm{~d} t^{2}+e^{2(c t / L)}\left[\mathrm{d} r^{2}+r^{2} \mathrm{~d} \Omega_{d-2}\right] .
$$

which can be be embedded into the $d+1$ dimensional flat spacetime

$$
\mathrm{d} s_{d+1}^{2}=\eta_{M N} \mathrm{~d} X^{M} \mathrm{~d} X^{N}=-\mathrm{d} X_{0}^{2}+\mathrm{d} X_{i}^{2}
$$

with $i=1,2, \ldots, d$. The vacuum Einstein equation associated with the $(d+1)$-dimensional flat metric (16) turns out to be $G_{M N}^{(d+1)}=0$.

Let us study the embedding of de-Sitter hypersurface in more details. It is a hyperbolid spacetime with radius $L$,

$$
L^{2}=-T^{2}+X_{i}^{2}, \quad \mathcal{N}^{M}=\frac{1}{L}\left(X_{0}, X_{i}\right)
$$

where $\mathcal{N}^{M}$ is the normal vector of the hypersurface pointing outwards. The cosmological constant $\Lambda=\frac{(d-1)(d-2)}{2 L^{2}}$ will play the role of the dark energy. Notice that to balance the Einstein equations with the induced de-Sitter metric $g_{\mu \nu}$ in (15), one requires either the cosmological constant or the apparent dark energy term.
Interestingly, for the pure de-Sitter spacetime (15), after considering (10) with extrinsic curvature $\mathcal{K}_{\mu v}=\frac{1}{L} g_{\mu v}$, the Brown-York stress tensor (12) turns out to be $\mathcal{T}_{\mu \nu}=$ $\overline{\mathcal{T}}_{\mu \nu}=-\frac{1}{\kappa_{d+1}} \frac{d-1}{L} g_{\mu \nu}$. Then we arrive at the stress tensor of apparent dark energy,

$$
\overline{\mathcal{T}}_{\mu \nu}=-\frac{\Lambda_{d}}{\kappa_{d}} g_{\mu \nu}, \quad \text { when } \frac{\kappa_{d+1}}{\kappa_{d}}=\frac{2 L}{d-2} .
$$

From (18) we read out the dark energy density formula

$$
\rho_{\Lambda}=\overline{\mathcal{T}}_{\mu \nu} \frac{u^{\mu} u^{v}}{c^{4}}=\frac{\Lambda_{d}}{\kappa_{d} c^{2}} .
$$

After considering (14) with $T=0$, we have the identity

$$
\frac{\overline{\mathcal{T}}^{2}}{d-1}-\overline{\mathcal{T}}^{\mu}{ }_{\nu} \overline{\mathcal{T}}^{v}{ }_{\mu}=-\frac{\rho_{\Lambda} c^{2}}{d-1} \overline{\mathcal{T}}
$$

Thus, assuming $\mathcal{T}_{\mu \nu}=\overline{\mathcal{T}}_{\mu \nu} \equiv-\frac{\Lambda}{\kappa_{d}} g_{\mu \nu}$ in the constraint equation (13), the pure de-Sitter spacetime satisfies the above identity automatically. Notice here the Brown-York stress tensor plays the role of dark energy and there is no matter or dark matter yet in the set-up.

Uniform Matter Perturbations. - Next we consider to add small amount of normal matters in with uniform distribution, such that we treat the de-Sitter metric as background. It describes dark energy dominated universe like today.

We consider that our university is uniform at large scale, and take the FLRM metric in $d$ dimension,

$$
\mathrm{d} s^{2}=-c^{2} \mathrm{~d} t^{2}+a(t)^{2}\left[\frac{\mathrm{d} r^{2}}{1-k r^{2}}+r^{2} \mathrm{~d} \Omega_{d-2}\right] .
$$

In the $\Lambda \mathrm{CDM}$ model, $k=0$, and

$$
H(a)^{2}=H_{0}^{2}\left[\Omega_{\Lambda}+\left(\Omega_{D}+\Omega_{B}\right) a^{-3}+\Omega_{R} a^{-4}\right]
$$

with $H(a) \equiv \frac{\dot{a}}{a}$, and $H_{0}$ is the Hubble constant today. Considering (18)(19), our assumption for the constraint relation (14) becomes

$$
\frac{\mathcal{T}^{2}}{d-1}-\mathcal{T}_{\mu \nu} \mathcal{T}^{\mu \nu}=-\frac{\rho_{\Lambda} c^{2}}{d-1}(T+\mathcal{T}) .
$$

This is the main constraint relation in this paper. Since in Einstein equation (8), $\mathcal{T}_{\mu \nu}$ is the Brown-York stress tensor playing the role of dark matter and dark energy, and $T_{\mu v}$ is the baryonic visible matter with mass density $\rho_{B} \ll \rho_{\Lambda}$. The baryonic matter and radiation are with energy density,

$$
T_{\mu \nu}^{B}=\rho_{B} u_{\mu} u_{v}, \quad T_{\mu \nu}^{R}=\rho_{R} u_{\mu} u_{v}+p_{R} h_{\mu v} .
$$

Now assume that the dark matter is induced with mass density $\rho_{D} \ll \rho_{\Lambda}$. The dark energy and cold dark matters are all assumed to be related to the extrinsic curvature.

$$
\mathcal{T}_{\mu \nu}=\mathcal{T}_{\mu \nu}^{\Lambda}+\mathcal{T}_{\mu \nu}^{D}, \quad T_{\mu \nu}=T_{\mu \nu}^{B}+T_{\mu \nu}^{R},
$$

where $\mathcal{T}_{\mu \nu}^{\Lambda}=-\left(\rho_{\Lambda} c^{2}\right) g_{\mu v}$ and $\mathcal{T}_{\mu \nu}^{D}=\rho_{D} u_{\mu} u_{v}$. Putting them back into the constraint equation (23), and subtracting equation (20), we arrive at,

$$
\rho_{D}^{2}=\frac{\rho_{\Lambda}}{d-2}\left[\rho_{D}-\rho_{B}-\rho_{R}+(d-1) \frac{p_{R}}{c^{2}}\right] .
$$


When $d=4$, the stress tensor of radiation is traceless $-\rho_{R} c^{2}+3 p_{R}=0$. If setting $\rho_{D} \simeq 5 \rho_{B}, \rho_{\Lambda} \simeq \rho_{c}-\rho_{D}-\rho_{B}$, we can recover Verlinde's constraint relation (4) approximately. Considering that the critical mass density of the university as $\rho_{c}=\frac{3 H_{0}^{2}}{8 \pi G}$, for which the spatial geometry is kept flat from the observation, then dividing both side of equation (26) by by $\rho_{c}^{2}$, we have

$$
\Omega_{D}^{2}=\frac{1}{2} \Omega_{\Lambda}\left(\Omega_{D}-\Omega_{B}\right) .
$$

If we further consider $\Omega_{\Lambda}+\Omega_{D}+\Omega_{B} \simeq 1$, then we derive our main result in (3),

$$
\Omega_{D}^{2}=\frac{1}{3}\left(\Omega_{D}-\Omega_{B}+\Omega_{B}^{2}\right)
$$

Considering $d=4, \Omega_{D} \simeq 5 \Omega_{B}$, as well as $\Omega_{B}=0.05 \ll 1$, we can also arrive at Verlinde's $\Omega_{D}^{2} \simeq \frac{4}{3} \Omega_{B}$ in (4).

On the other hand, since $\Omega_{B}+\Omega_{D} \lesssim \Omega_{\Lambda}$, although not so precise, our de sitter background is still a good approximation. However, if we consider the dark matter in smaller scales around the galaxies and compare with rotational curves, we need to consider the effects of backreaction of the normal matters, with new derivations.

Constraints from Gravitational Waves. - Recently it is argued that two relativistic models of modified Newtonian dynamics seem inconsistent with observations [5]. Modified gravity models face constraints from two aspects: one is the constraint of the energy loss rate from ultra high energy cosmic rays, which indicates that gravitational waves should propagate at the speed of light; the other is the observed gravitational waveforms from LIGO, which are consistent with Einstein's gravity and suggest that the gravitational wave should satisfy linear equations of motion in the weak-field limit.

Although E. Verlinde suggested similar modifications of Newtonian dynamics as in MOND theories [2], which emerges with different underlining physical origin, there is no covariant equations of motion for the gravitational waves. While for our toy model in the previous sections, we have shown that the Einstein equations are not necessarily modified in our scenarios. The induced dark sectors play the same role as that in the $\Lambda \mathrm{CDM}$ models, so it can pass the above mentioned constraints [5]. To be more specific, the extra apparent dark matter as extra terms in Einstein equation fills the space as dark medium and modifies the the speed of the light. In our induced gravity, gravitational field equation is

$$
\mathcal{R}_{\mu \nu}-\frac{1}{2} g_{\mu \nu} \mathcal{R}=\frac{8 \pi G}{c^{4}}\left(T_{\mu \nu}+\mathcal{T}_{\mu \nu}\right)
$$

which leads to $0=\nabla^{\mu} G_{\mu v}=\nabla^{\mu} T_{\mu \nu}+\nabla^{\mu} \mathcal{T}_{\mu \nu}$. Thus it is similar to the effects of cold dark matter, and it does not conflict with the observations from LIGO so far.

The dark matter scenarios provide one extra effect on top of the extra dimensional modification in our setups. However, if we indeed take a higher dimensional point of views [6, 7], we expect one extra breezing mode on top of two polarized propagating modes. The extra breezing mode is constraint by the current experiments. There are also multiple massive Kaluza-Klein gravitational modes associated with the extra dimensions. Although those massive modes decay fast and may not reach the gravitational waves detector, they are constrained as well by the gravitational waves signal templates from the binary black hole signals.

\section{Connection with other Scenarios}

We compare our approach with two well studied scenarios here. One is the holographic scenario of our universe, and the other is the brane world models. Naively they look similar, in a way that they all embed our universe into higher dimensional spacetime. However, the holographic models is a dual equivalent description, while in the brane world models, there exist real extra dimensions.

Holographic Universe. - Let us first compare with the holographic universe scenario. There is an effective contribution from the holographic stress tensor, which can be identified as the stress tensor of dark energy and dark matter. Let us start with the Einstein-Hilbert action in $(d+$ 1)-dimension, with the Gibbons-Hawking-York boundary term

$$
\begin{aligned}
S_{d+1}= & \frac{1}{2 \kappa_{d+1}} \int_{\mathcal{M}} \mathrm{d}^{d+1} x \sqrt{-\tilde{g}}\left(R_{d+1}-2 \Lambda_{d+1}\right) \\
& +\frac{1}{\kappa_{d+1}} \int_{\partial \mathcal{M}} \mathrm{d}^{d} x \sqrt{-g} \mathcal{K}_{d},
\end{aligned}
$$

with $\tilde{g}_{M N}$ being the metric in $d+1$ dimension. After the variation, we have

$$
\begin{aligned}
\left(2 \kappa_{d+1}\right) \delta S_{d+1} & =\left[R_{d+1}^{M N}-\frac{1}{2} R_{d+1} \tilde{g}^{M N}+\Lambda_{d+1} \tilde{g}^{M N}\right] \delta \tilde{g}_{M N} \\
& +2\left(\mathcal{K}^{\mu \nu}-\mathcal{K} g^{\mu \nu}\right) \delta g_{\mu \nu} .
\end{aligned}
$$

$\mathcal{K}_{\mu \nu}$ is the external curvature of the time-like brane, which motivates our Brown-York stress tensor in (12). In the usual paradigm of holography, we do not consider the back reaction of the holographic stress tensor on the hypersurface. In our toy model which modifies the Einstein equations into (1), we do consider it.

In our toy model, the holographic screen is embedded in one higher dimensional flat spacetime, which is quite similar to the holography in Rindler spacetime. It is one successful realization of holography in flat spacetime, where the bulk background is flat spacetime, and the induced Brown-York stress tensor gives the dual Rindler fluid [8]. Rindler fluid has the energy density $\rho_{U}=0$, and pressure $p_{U}=a$, where $a$ is the acceleration of the Rindler observer. The vacuum Einstein equation also yields the equation of states $p_{U}=T_{U} s$, with $T_{U}$ being the Unruh temperature, and $\mathrm{s}$ being the Bekenstein entropy density of the horizon. Especially, it is found that the Petrov Type I conditions give rise to additional constraint relation of the holographic stress tensor. [9]. It is expected to be generalized to our case as another candidate of constraint equations that is similar to (13).

Mathematically, the de-Sitter metric (15) or the more general FRW metric (21) can also be embedded into the one higher dimensional AdS spacetime, which leads to the holographic models of the universe. Although the holographic correspondence is more clear there, one needs to deal with another problem on the origin of the cosmological constant. That is one of the reasons that we choose the 
flat embedding in this paper instead of the AdS. The latter one is interesting for further study.

What is more, it is natural to consider that the entanglement between two cosmological horizons may have an impact on the gravity as suggested by Verlinde [2]. In the $4+1$ dimensional space time, it is more clear to see this relation through the embeddings of wormholes. It is show in [10], that the EPR pair in $3+1$ can be described by the worm hole in 4+1 dimensional AdS spacetime.

Brane World Scenario. - Now let us compare our model to the well studied brane world models. Except for the constraint equations, we also have the dynamical Einstein equation in higher dimensions. We analyze the equations induced on the hypersurface

$$
\begin{aligned}
R_{\mu \nu}^{(d)} & =\left(\mathcal{K} g_{\mu \sigma}-\mathcal{K}_{\mu \sigma}\right) \mathcal{K}_{v}^{\sigma}+\mathcal{M}_{\mu \nu}, \\
\mathcal{M}_{\mu \nu} & \equiv g_{\mu}{ }^{M} g_{\nu}{ }^{N} R_{M N}^{(d+1)}+g_{\mu}{ }^{M} \mathcal{N}^{P} g_{v}{ }^{N} \mathcal{N}^{Q} R_{M P N Q}^{(d+1)}
\end{aligned}
$$

Although $R_{M N}^{(d+1)}=0$ in flat spacetime, it is not necessary true for $R_{M P N Q}^{(d+1)}$, which depends on the coordinate choices. After including the normal matters $T_{\mu \nu}$, in principle we can also define the effective stress tensor from brane world models

$$
\begin{gathered}
R_{\mu \nu}-\frac{1}{2} R g_{\mu \nu}=T_{\mu \nu}+\mathcal{T}_{\mu \nu}^{\mathcal{M}} \\
\mathcal{T}_{\mu \nu}^{\mathcal{M}} \equiv\left(\mathcal{K}_{\mu \sigma}-\mathcal{K}_{\mu \sigma}\right) \mathcal{K}_{\nu}^{\sigma}+\mathcal{W}_{\mu \nu}-\frac{1}{2}\left(\mathcal{K}^{2}-\mathcal{K}_{\rho \sigma} \mathcal{K}^{\rho \sigma}\right) g_{\mu \nu}
\end{gathered}
$$

which includes the description of the the hyper surface evolution. In the de Sitter spacetime, $\mathcal{K}_{\mu \sigma}=\frac{1}{L} g_{\mu \nu}$ leads to $\mathcal{T}_{\mu \nu}^{\mathcal{M}}=-\Lambda g_{\mu \nu}$, and $\mathcal{W}_{\mu \nu}$ is associated with the bulk Weyl tensor. However, we have tried the perturbation based on this formula, but the constraint relations is not as good as our equation (3).

Verlinde's Emergent Gravity. - Following Verlinde's model for apparent dark matter, there seem to be three crucial conditions for his construction so far. First, there is the background entropy, that is what he calls the elastic medium occupying the $(3+1)$-dimensional spacetime. Second, the positive cosmological constant, provides the thermal bath with the Gibbons-Hawking temperature $H / 2 \pi$. The last, the apparent dark matter is only the response to the presence of the normal matters.

The braneworld scenarios can offer similar above mentioned conditions and become a natural playground for Verlinde's emergent gravity. The elastic medium full of entropy can be explained from higher dimensions, by treating our $(3+1)$ dimensional spacetime as the boundary of the bulk theory. Cosmological constant and the standard model can be easily implemented with braneworld in many literatures [11].Actually that is one of main motivations that braneworld was widely discussed before. Most interestingly, the branes with tensions and dynamics, can react to the matter fields we put in, with extra terms we introduced in the last section. Especially the exterior curvature, a concept valid only from higher dimensional spacetime, describes the "elastic response" nature of the apparent dark matter from Verlinde's theory.
Summary. - In summary, We give a new viewpoint on the dark components of our universe, which originates from the induced stress tensor of higher dimensional flat spacetime. There are only normal matters on the brane, and the dark energy and dark matter are considered as the response of normal matters due to the geometric effects.

In our approaches, the toy and the more developed ones, partly borrowed from the braneworld scenario and combined with holographic universe models, which resembles Verlinde's emergent gravity in a subtle way. We do have a covariant relativistic form of Lagrangian. Our ansatz produces the late universe component data from $\Lambda \mathrm{CDM}$ model, and is also expected to be consistent with early universe evolution from its braneworld like features, although we have not produced the CMB prediction of our scenarios in this note. We are going to study this CMB prediction in the near future.

From our observation, it is still quite interesting to ask whether there are real extra dimensions, although there is no evidence from experiments so far. One recent study may come from gravitational waves physics in [7], in addition to the long searching constraints from colliders and precision measurements of gravity.

Acknowledgements - S. Sun thanks the supported from NCTS for the conference. We thank R. G. Cai, H. Tye, S. J. Wang for helpful conversions.

\section{References}

[1] E. P. Verlinde, JHEP 1104, 029 (2011) [arXiv:1001.0785].

[2] E. P. Verlinde, SciPost Phys. 2, 016 (2017) [arXiv:1611.02269 [hep-th]].

[3] P. A. R. Ade et al. [Planck Collaboration], Astron. Astrophys. 594, A13 (2016) [arXiv:1502.01589 [astro-ph.CO]].

[4] J. D. Brown and J. W. York, Jr., Phys. Rev. D 47, 1407 (1993) [gr-qc/9209012].

[5] P. M. Chesler and A. Loeb, Phys. Rev. Lett. 119, 031102 (2017) [arXiv:1704.05116 [astro-ph.HE]].

[6] T. Kobayashi and T. Tanaka, Phys. Rev. D 73, 044005 (2006) [hep-th/0511186].

[7] D. Andriot and G. Lucena Gómez, JCAP 1706, 048 [arXiv:1704.07392 [hep-th]].

[8] I. Bredberg, C. Keeler, V. Lysov and A. Strominger, JHEP 1207, 146 (2012) [arXiv:1101.2451 [hep-th]].

[9] V. Lysov and A. Strominger, arXiv:1104.5502 [hep-th]. R. G. Cai, L. Li, Q. Yang and Y. L. Zhang, JHEP 1304, 118 (2013) [arXiv:1302.2016 [hep-th]]. R. G. Cai, Q. Yang and Y. L. Zhang, Phys. Rev. D 90, 041901 (2014) [arXiv:1401.7792 [hep-th]].

[10] K. Jensen and A. Karch, Phys. Rev. Lett. 111, 211602 (2013) [arXiv:1307.1132]. J. Sonner, Phys. Rev. Lett. 111, 211603 (2013) [arXiv:1307.6850]. J. W. Chen, S. Sun and Y. L. Zhang, arXiv:1612.09513 [hep-th].

[11] J. Frieman, M. Turner and D. Huterer, Ann. Rev. Astron. Astrophys. 46, 385 (2008) [arXiv:0803.0982 [astroph]]. G. R. Dvali, G. Gabadadze and M. Porrati, Phys. Lett. B 485, 208 (2000) [hep-th/0005016]. D. B. Kaplan and S. Sun, Phys. Rev. Lett. 108, 181807 (2012) [arXiv:1112.0302 [hep-ph]]. 